\title{
Les nanocristaux semi-conducteurs ou boîtes quantiques
}

\author{
D. Ricard ${ }^{1}$ \\ Laboratoire d'Optique Quantique, École Polytechnique, 91128 Palaiseau cedex, \\ France
}

\begin{abstract}
Résumé : Nous discutons ici des propriétés optiques linéaires et non linéaires de colloïdes ou de verres contenant des nanocristaux semi-conducteurs, l'exemple choisi étant CdSe. Le confinement des porteurs conduit à un spectre de raies élargies surtout par la grande dispersion de taille des nanocristaux. La non-linéarité qui intéresse les applications est l'effet Kerr statique ou optique. Dans ce dernier cas, il faut distinguer le régime résonant où l'effet dominant est un effet de population et le régime non résonant où le déplacement radiatif est à l'origine de l'indice non linéaire. L'absorption a deux photons est également considérée.
\end{abstract}

\section{INTRODUCTION}

Bien que les colloïdes métalliques par exemple soient connus depuis fort longtemps, l'étude systématique des systèmes mésoscopiques a commencé il y a une vingtaine d'années. Dans ces systèmes, de taille intermédiaire entre le microscopique et le macroscopique, le mouvement des porteurs, électrons ou trous, est limité par des barrières de potentiel. Il en résulte une quantification de l'énergie cinétique selon le schéma bien connu du puits de potentiel. Ce genre d'étude a d'abord porté sur les puits quantiques formés d'un matériau semi-conducteur de bande interdite plus faible et d'épaisseur de quelques nanomètres pris en sandwich entre deux "barrières" formées d'un autre matériau semi-conducteur de bande interdite plus grande. L'exemple le plus classique est formé à partir du couple $\mathrm{GaAs} / \mathrm{Al}_{\mathbf{x}} \mathrm{Ga}_{1-\mathrm{x}} \mathrm{As}$. Ces puits quantiques possèdent des propriétés remarquables telles que la présence de pics excitoniques même à température ambiante. Cela leur confere des propriétés optiques non linéaires très intéressantes.

Dans un puits quantique, le mouvement des porteurs est confiné dans une dimension mais reste libre dans les deux autres dimensions. C'est pourquoi on les appelle parfois des matériaux quasi 2D. Étant données les propriétés de ces matériaux quasi 2D, on s'est demandé ce qu'il en serait des fils quantiques avec confinement dans deux dimensions, matériaux quasi 1D, ou des points ou boîtes quantiques, matériaux quasi OD. Pour des raisons technologiques de fabrication, les boîtes quantiques, qui sont donc de petits cristaux métalliques ou semiconducteurs, ont été étudiées en premier. Il est en effet facile de préparer des colloïdes d'or ou d'argent par réaction chimique en milieu aqueux et les verres contenant des nanoparticules de

\footnotetext{
' Nouvelle adresse : Laboratoire Aimé Cotton, UPR 3321 du CNRS, Centre Scientifique d'Orsay, bâtiment 505, 91405 Orsay cedex, France.
} 
ces métaux sont utilisés depuis des siècles dans l'art du vitrail. On sait également fabriquer des colloïdes de matériaux II-VI tels que CdS, toujours par réaction chimique. Les techniques s'étant affinées, on sait maintenant contrôler la taille de ces nanocristaux et aussi préparer des colloïdes de matériaux III-V. On sait également protéger la surface des particules en y attachant des molécules organiques. On peut enfin préparer des verres contenant des particules de matériaux II-VI ou I-VII en mélangeant les composants du semi-conducteur (Cd et Se par exemple) au verre à haute température. Le verre en fusion est alors refroidi rapidement. On procède enfin à la croissance des cristaux par traitement thermique à une température de 500 à $700^{\circ} \mathrm{C}$. La taille moyenne est déterminée par la température et la durée du traitement.

\section{MATERIAUX COMPOSITES ET EFFET DE CHAMP LOCAL}

Nous discutons donc ici les propriétés de nanocristaux métalliques ou (et surtout) semiconducteurs, en particulier leurs propriétés optiques non linéaires. Ces nanocristaux sont nécessairement dispersés dans un milieu diélectrique (verre, eau...) et l'ensemble forme un matériau dit composite. De par la présence de l'interface entre nanocristaux et milieu hôte, un matériau composite possède des propriétés différentes de celles de ses composants. Par exemple, un verre dopé par des nanoparticules d'or présente un pic d'absorption dans le vert.

Ceci est dû à un effet de champ local. Les deux composants ont des permittivités différentes, $\varepsilon$ pour les cristallites et $\varepsilon_{\mathrm{d}}$ pour la matrice diélectrique. Il en résulte une différence entre le champ électrique à l'intérieur d'une particule $\mathrm{E}_{\mathrm{in}}$ et le champ électrique régnant dans le milieu environnant E. Pour des particules sphériques de taille petite devant la longueur d'onde laser, on a la relation simple [1]

$$
\mathbf{E}_{\text {in }}=\frac{3 \varepsilon_{\mathrm{d}}}{\varepsilon+2 \varepsilon_{\mathrm{d}}} \mathrm{E}
$$

Le facteur $f=3 \varepsilon_{d} /\left(\varepsilon+2 \varepsilon_{d}\right)$ joue le rôle d'un facteur de champ local macroscopique. Il dépend en général de la fréquence puisque $\varepsilon$ en dépend (alors que $\varepsilon_{\mathrm{d}}$ est constant en bonne approximation). En particulier, s'il existe une fréquence pour laquelle la partie réelle du dénominateur $s$ 'annule, alors le facteur $f$ présente une résonance appelée résonance plasma de surface. Le coefficient d'absorption contient le facteur $f$ en module au carré et présente donc lui aussi une résonance. Ainsi s'explique le pic d'absorption vers $530 \mathrm{~nm}$ pour un verre dopé par des particules d'or [2]. Pour cette fréquence, les lignes de champ semblent attirées vers l'intérieur de la sphère. On parle de confinement diélectrique.

Mais cet effet n'est réellement important que pour les métaux comme l'or ou l'argent. Il peut néanmoins avoir une conséquence importante. En effet, le champ interne $\mathrm{E}_{\mathrm{in}}$ dépend de $\varepsilon$ selon l'équation (1). Mais, par l'effet Kerr optique, $\varepsilon_{\mathrm{d}}$ dépend à son tour du champ interne. Cette inter-relation peut conduire au phénomène de bistabilité intrinsèque, c'est-à-dire sans cavité Fabry-Perot. Pour certaines valeurs de l'intensité incidente, donc de E, l'absorbance de l'échantillon (par l'intermédiaire du facteur de champ local) peut prendre deux valeurs différentes dépendant de l'histoire précédente de E. L'effet Kerr optique peut être très important pour un cristallite semi-conducteur de CdS par exemple. Mais le facteur de champ local reste faible pour une sphère de CdS et la bistabilité n'est alors pas observable. On peut contourner la difficulté en fabriquant des nanosphères de $\mathrm{CdS}$ enrobées d'une coquille d'argent. On combine alors la forte non-linéarité de CdS avec la résonance plasma de surface 
de l'argent (le calcul du facteur de champ local est alors plus complexe) et on peut observer le phênomène de bistabilité intrinsèque [3].

Pour les semi-conducteurs cependant, même au voisinage des niveaux discrets dont nous parlerons ci-dessous, la dispersion de la permittivité reste négligeable et le facteur de champ local est pratiquement une constante d'ailleurs inférieure à un. Nous ne nous en soucierons donc que peu. Si l'on veut néanmoins comparer la non-linéarité d'un nanocristal à celle du cristal massif, il est nécessaire de savoir relier la susceptibilité $\tilde{\chi}^{(3)}$ du composite à celle de ses constituants. L'expression est la suivante :

$$
\tilde{\chi}^{(3)} \approx \chi_{\mathrm{d}}^{(3)}+p \mathrm{f}^{4} \chi^{(3)}
$$

expression valable lorsque la fraction volumique $p$ occupée par les nanocristaux est petite devant 1. $\chi^{(3)}$ est la susceptibilité du nanocristal et $\chi_{\mathrm{d}}^{(3)}$ celle du matériau hôte. En régime résonant, i.e. quand la fréquence laser correspond à une fréquence de transition des nanocristaux, la contribution de la matrice hôte est négligeable. Il n'en est plus de même hors résonance.

\section{LE CONFINEMENT ÉLECTRONIQUE}

L'effet de champ local étant relativement peu important pour les nanocristaux semi-conducteurs sur lesquels nous nous concentrons, l'effet dominant dans la différence de comportement entre boîtes quantiques et matériaux massifs provient du confinement (du mouvement) électronique. Nous commençons par discuter les conséquences de ce confinement électronique sur le spectre des niveaux d'énergie qu'un électron peut occuper dans un tel nanocristal.

\subsection{Les différents régimes de confinement}

Pour un semi-conducteur intrinsèque, l'état fondamental correspond à une bande de valence pleine et à une bande de conduction vide. Les premiers états excités s'obtiennent en faisant passer un électron du haut de la bande de valence au bas de la bande de conduction. On crée ainsi une paire électron-trou. En se limitant à deux bandes paraboliques et en utilisant l'approximation de la masse effective, l'équation aux valeurs propres pour une telle paire s'écrit

$$
\left(-\frac{\hbar^{2}}{2 m_{e}} \Delta_{e}+V\left(r_{e}\right)-\frac{\hbar^{2}}{2 m_{h}} \Delta_{h}+V\left(r_{h}\right)-\frac{e^{2}}{\varepsilon r_{e-h}}\right) \phi\left(\mathbf{r}_{e}, \mathbf{r}_{h}\right)=E^{e-h} \phi\left(r_{e}, r_{h}\right)
$$

où $\Delta$ est le Laplacien, $m_{e}$ et $m_{h}$ les masses effectives de l'électron et du trou, $e^{2}=q^{2 / 4 \pi \varepsilon_{0}}$ ( $q$ étant la charge élémentaire et $\varepsilon_{0}$ la permittivité du vide. $V$ est le potentiel de confinement supposé constant à l'intérieur de la particule et infini à l'extérieur, $\phi$ est la fonction d'onde enveloppe et $\mathrm{E}^{\mathrm{e}-\mathrm{h}}$ l'énergie propre. Le dernier terme dans le Hamiltonien représente l'énergie d'interaction coulombienne entre électron et trou, $\mathrm{r}_{\mathrm{e}-\mathrm{h}}$ étant la distance électron-trou.

L'équation (3) n'est pas soluble analytiquement mais elle se simplifie dans deux cas limites définis par rapport aux échelles de taille pertinentes [4]. Deux énergies apparaissent 
naturellement, l'énergie cinétique de confinement qui varie en $1 / \mathrm{R}^{2}$ si on suppose les particules sphériques de rayon $R$ et l'énergie d'interaction coulombienne qui varie en $1 / R$. Les échelles de taille pertinentes sont le rayon $R$ du nanocristal et le rayon de Bohr de l'exciton $a_{B}=\varepsilon \hbar^{2} / \mu e^{2}$ où $\mu$ est la masse réduite $\mu=m_{e} m_{h} /\left(m_{e}+m_{h}\right)$. On montre que, si $R \gg a_{B}$, l'énergie d'interaction coulombienne domine et on peut négliger, en première approximation, les termes en V dans le Hamiltonien de l'équation (3). On retrouve alors le cas d'un système hydrogénoïde, l'exciton de Wannier, qui existe comme dans le semi-conducteur massif. On peut ensuite, en deuxième approximation, tenir compte de la taille finie de la particule. L'exciton est confiné avec une énergie de confinement faible de l'ordre de $\hbar^{2} / \mathrm{MR}^{2}$ où $\mathrm{M}$ est la masse totale $m_{e}+m_{h}$. On est dans le régime dit de confinement faible.

Dans le cas limite inverse où $R \ll a_{B}$, c'est l'énergie cinétique de confinement qui domine. On peut alors négliger, en première approximation, le dernier terme du Hamiltonien de l'équation (3). Ce Hamiltonien se réduit alors à la somme de deux Hamiltoniens indépendants, l'un pour l'électron, l'autre pour le trou ; le mouvement des deux particules est alors découplé. Ce régime est celui du confinement fort. L'expérience montre que les conditions requises pour être dans l'un de ces deux régimes sont moins draconiennes qu'indiqué cidessus [5]. Si $R>3 \mathrm{a}_{\mathrm{B}}$, on est en régime de confinement faible. C'est en général le cas pour les nanocristaux de $\mathrm{CuCl}$ pour lequel $\mathrm{a}_{\mathrm{B}} \approx 0,7 \mathrm{~nm}$. Si $\mathrm{R}<\mathrm{a}_{\mathrm{B}}$, on est en régime de confinement fort, situation assez facile à obtenir pour CdSe pour lequel $a_{B} \approx 5,6 \mathrm{~nm}$. Nous nous limitons dans la suite à ce régime de confinement fort en prenant le plus souvent pour exemple les nanocristaux de CdSe. Ce cas s'appliquerait aussi aux matériaux III-V.

\subsection{Le confinement fort}

En régime de confinement fort, l'électron et le trou sont donc confinés indépendamment. La fonction d'onde enveloppe de l'électron $\varphi^{e}(\mathbf{r})$ par exemple obéit à l'équation aux valeurs propres

$$
\left(-\frac{\hbar^{2}}{2 m_{e}} \Delta+V(r)\right) \varphi^{e}(\mathbf{r})=E^{e} \varphi^{e}(\mathbf{r})
$$

C'est le problème simple de la particule libre dans une boîte. Pour une boîte sphérique, les solutions sont de la forme

$$
\varphi_{l \mathrm{mn}}(\mathbf{r})=\mathrm{B}_{l \mathrm{n}} \mathrm{j}_{l}\left(\alpha_{l \mathrm{n}} \frac{\mathrm{r}}{\mathrm{R}}\right) \mathrm{Y}_{l}^{\mathrm{m}}(\theta, \varphi)
$$

où les $\mathrm{Y}_{l}^{\mathrm{m}}$ sont les harmoniques sphériques, $\mathrm{j}_{l}(\mathrm{x})$ est la fonction de Bessel sphérique d'ordre $l, \alpha_{l \mathrm{n}}$ son $\mathrm{n}^{\mathrm{i} i \mathrm{eme}}$ zéro et $\mathrm{B}_{l \mathrm{n}}$ une constante de normalisation. $\mathrm{r}, \theta$ et $\varphi$ sont les coordonnées sphériques de r. Prenant l'origine des énergies au sommet de la bande de valence, l'énergie propre correspondante est 


$$
\mathrm{E}_{\mathrm{ln}}^{\mathrm{e}}=\mathrm{E}_{\mathrm{g}}+\frac{\hbar^{2} \alpha_{l \mathrm{n}}^{2}}{2 \mathrm{~m}_{\mathrm{e}} \mathrm{R}^{2}}
$$

où $E_{\mathrm{g}}$ est la largeur de la bande interdite du semi-conducteur massif. Au lieu d'avoir un quasicontinuum de niveaux comme dans le cas du matériau massif (cas que l'on retrouve en faisant tendre $R$ vers l'infini), on a maintenant un ensemble de niveaux discrets. Les premiers niveaux sont $1 \mathrm{~s}$ avec $l=0, \mathrm{n}=1$ et $\alpha_{01}=\pi$, puis $1 \mathrm{p}$ avec $l=1, \mathrm{n}=1$ et $\alpha_{11}=4,49$, puis $1 \mathrm{~d}, 2 \mathrm{~s}$, etc. Rappelons que la fonction d'onde totale de l'électron est

$$
\psi_{l \mathrm{mn}}^{\mathrm{e}}\left(\mathbf{r}_{\mathrm{e}}\right)=\varphi_{l \mathrm{mn}}\left(\mathbf{r}_{\mathrm{e}}\right) \mathrm{u}_{\mathrm{c}}\left(\mathbf{r}_{\mathrm{e}}\right)
$$

où $\mathrm{u}_{\mathrm{c}}$ est la partie périodique correspondant à la bande de conduction.

Si nous supposons que la bande de valence est non dégénérée, la fonction d'onde enveloppe pour le trou obéit à une équation similaire dont les fonctions propres sont les mêmes que pour l'électron. L'énergie propre correspondante s'écrit

$$
\mathrm{E}_{l \mathrm{n}}^{\mathrm{h}}=\frac{\hbar^{2} \alpha_{l \mathrm{n}}^{2}}{2 \mathrm{~m}_{\mathrm{h}} \mathrm{R}^{2}}
$$

Là encore, la fonction d'onde totale est le produit de la fonction enveloppe par une partie périodique $\mathbf{u}_{\mathbf{v}}\left(\mathbf{r}_{\mathbf{h}}\right)$.

Après avoir traité séparément le mouvement de l'électron et du trou, on peut ensuite, en deuxième approximation, tenir compte de l'interaction coulombienne par la théorie des perturbations.

\subsection{Discussion des approximations}

Dans le traitement précédent, un certain nombre d'hypothèses ont été faites. D'abord, la boîte a été supposée sphérique. Le calcul est également faisable pour une boîte parallélépipédique. Le cas de particules ellipsoïdales s'écartant peu de la forme sphérique a aussi été considéré [6]. Les conclusions générales, niveaux discrets et énergie de confinement, dépendent peu de la forme des nanocristaux. La non-sphéricité peut par contre avoir des conséquences sur la structure fine des niveaux. Nous y reviendrons dans la section 4.3. Nous avons ensuite supposé les bandes paraboliques. L'énergie de confinement étant inversement proportionnelle à la masse effective, cf. équations (6) et (8), et la masse effective de l'électron étant plus petite, la non-parabolicité se manifeste surtout pour la bande de conduction. On peut en tenir compte [7] ce qui a pour effet de diminuer l'énergie de confinement.

Nous avons aussi supposé une barrière de confinement infiniment haute qui impose à la fonction d'onde enveloppe de s'annuler à l'interface. En réalité, la barrière de potentiel est d'environ $2 \mathrm{eV}$. Avec une barrière finie, la fonction d'onde est légèrement plus étendue et l'énergie de confinement par conséquent plus faible. Pour écrire l'expression de l'énergie d'interaction coulombienne entre électron et trou, dernier terme du Hamiltonien dans l'équation (3), nous avons encore supposé que nanocristal et matrice hôte ont la même permittivité. En réalité, cette énergie d'interaction coulombienne est la somme du dernier terme de notre 
Hamiltonien et d'une énergie dite de polarisation. Le problème a été traité par une méthode variationnelle [8] et par perturbation [9]. Il a pour effet de déformer légèrement les fonctions d'onde enveloppes.

Nous avons aussi utilisé l'approximation de la masse effective qui est valable lorsque la fonction d'onde enveloppe varie peu à l'échelle de la cellule élémentaire. Cette approche de type physique des solides revient donc à supposer que le nanocristal est assez « gros », que le nombre d'atomes internes l'emporte largement sur celui des atomes en surface. Cela demande des nanocristaux contenant plusieurs centaines d'atomes. En fait, un nanocristal est un objet intermédiaire entre une molécule et un cristal. Si le nanocristal est assez "gros », l'approche précédente doit être valable. S'il est très petit, une approche du type orbitales moléculaires (par exemple LCAO) doit être utilisée. De tels calculs ont été faits, en général en supposant une hybridation $\mathrm{sp}^{3}[10]$. On peut voir ainsi se former progressivement les bords des bandes de valence et de conduction.

Nous avons enfin supposé que les bandes sont non dégénérées. Pour la bande de conduction issue d'orbitales atomiques de type $s$, hormis la dégénérescence de spin, l'hypothèse est justifiée. La bande de valence est issue d'orbitales atomiques de type p et est donc, si l'on tient compte du spin, dégénérée 6 fois. Le couplage spin-orbite lève en partie la dégénérescence en conduisant à un moment cinétique atomique $\mathrm{J}$ égal à $3 / 2$ pour les bandes de trous lourds et de trous légers ou à $1 / 2$ pour la bande décalée. Ceci est valable pour le cristal massif et le reste pour les puits quantiques. Pour une particule sphérique par contre, à cause de la géométrie sphérique du potentiel de confinement, il y a couplage entre le moment cinétique J et celui $\mathbf{L}$ de la fonction d'onde enveloppe. Le problème a été traité par analogie avec celui des états accepteurs [11]. Le bon nombre quantique est le moment cinétique total F. Il y a mélange des bandes de valence et mélange des fonctions d'onde enveloppes [7]. Considérant les premiers niveaux pour lesquels $F=3 / 2$, on peut avoir des fonctions enveloppes paires impliquant $l=0$ et $l=2$ ou impaires impliquant $l=1$ et $l=3$. Les niveaux correspondants sont notés $\mathrm{nS}_{3 / 2}$ et $\mathrm{nP}_{3 / 2}$. Les états de l'électron sont inchangés mais sont en général notés $1 \mathrm{~S}_{\mathrm{e}}$, $1 \mathrm{P}_{\mathrm{e}} \ldots$ au lieu de $1 \mathrm{~s}, 1 \mathrm{p} .$.

\section{PROPRIÉTÉS LINÉAIRES}

Les transitions permises sont celles pour lesquelles l'élément de matrice de transition est non nul. Nous nous plaçons d'abord dans le cadre de l'approche simplifiée de la section 3.2 et nous nous demandons quelle est la probabilité de faire passer un électron de l'état $(l, \mathrm{~m}, \mathrm{n})$ de la bande de valence à l'état $\left(l^{\prime}, \mathrm{m}^{\prime}, \mathrm{n}\right.$ ') de la bande de conduction. Les fonctions d'onde étant composées d'une partie lentement variable $\varphi_{l \mathrm{mn}}$ et d'une partie plus rapidement variable $u_{c}$ ou $\mathrm{u}_{\mathrm{v}}$ et l'opérateur quantité de mouvement $\mathrm{p}$ étant $\hbar / \mathrm{i}$ fois l'opérateur gradient, l'élément de matrice se simplifie

$$
\left\langle\psi_{l m^{\prime} n^{\prime}}^{\mathrm{e}}|\mathrm{p}| \psi_{l \mathrm{mn}}^{\mathrm{h}}\right\rangle \approx\left\langle\mathrm{u}_{\mathrm{c}}|\mathrm{p}| \mathrm{u}_{\mathrm{v}}\right\rangle \int_{\text {sphère }} \varphi_{l^{\prime} \mathrm{m}^{\prime} \mathrm{n}^{\prime}}^{*}(\mathbf{r}) \varphi_{l \mathrm{mn}}(\mathbf{r}) \mathrm{d}^{3} \mathrm{r}
$$

Il est le produit de ce que l'on nomme habituellement $\mathrm{p}_{\mathfrak{c v}}$ par l'intégrale de recouvrement des fonctions d'onde enveloppes pour l'électron et le trou.

Or les $\varphi_{l \mathrm{mn}}$ forment une base orthonormée. L'intégrale de recouvrement est donc simplement $\delta_{l l} \delta_{\mathrm{mm}} \delta_{\mathrm{nn}}$ où $\delta_{\mathrm{ij}}$ est le symbole de Kronecker. Les seules transitions interbandes per- 
mises sont celles qui conservent les nombres quantiques $l, \mathrm{~m}$ et $\mathrm{n}$, c'est-à-dire les transitions $1 \mathrm{~s}-1 \mathrm{~s}, 1 \mathrm{p}-1 \mathrm{p} .$. Une telle transition se produit à la fréquence $\omega_{l n}$ donnée par

$$
\hbar \omega_{l \mathrm{n}}=\mathrm{E}_{\mathrm{g}}+\frac{\hbar^{2} \alpha_{l \mathrm{n}}^{2}}{2 \mu \mathrm{R}^{2}}-\beta \frac{\mathrm{e}^{2}}{\varepsilon \mathrm{R}}
$$

Le deuxième terme est l'énergie cinétique de confinement et le troisième l'énergie d'interaction coulombienne. $\beta$ est un nombre voisin de 1,8 pour la transition $1 \mathrm{~s}-1 \mathrm{~s}$. La dégénérescence de la bande de valence modifie un peu ces résultats. Par exemple les transitions sont permises de tous les $n S_{3 / 2}$ vers $1 S_{e}$ mais la plus intense est la transition $1 S_{3 / 2}-1 S_{e}$.

\subsection{Spectres d'absorption et de luminescence}

À partir des considérations précédentes, nous nous attendons à un spectre d'absorption fait de raies, la fréquence de la première transition étant d'autant plus décalée vers le bleu que le nanocristal est petit. La figure 1 montre les spectres d'absorption à basse température de trois verres dopés par nanocristaux de CdSe dont les rayons moyens sont de 3,8 nm, 2,6 nm et 2,1 $\mathrm{nm}$. Le décalage vers le bleu est visible sur le spectre lui-même. La dérivée seconde du spectre fait plus clairement apparaître les raies attendues, même si elles sont élargies. Les deux premières sous-structures correspondent aux transitions $1 S_{3 / 2}-1 S_{e}$ et $2 S_{3 / 2}-1 S_{e}$. Vient ensuite généralement la transition $1 P_{3 / 2}-1 P_{e}$, mais cela dépend de la taille des particules.

Les spectres de la figure 1 ont été obtenus pour des échantillons de très bonne qualité. En général, l'élargissement des raies fait que la sous-structure de la première bande n'est pas résolue, même à basse température. Lorsque nous traiterons des propriétés non linéaires en régime résonant, la résonance concernera cette bande. Nous la nommerons alors du terme vague de bande $1 \mathrm{~s}-1 \mathrm{~s}$ ou de première bande.

La partie supérieure de la figure 2 montre une partie du spectre de luminescence d'un colloïde de CdSe [12]. On observe un pic relativement étroit légèrement décalé vers le rouge par rapport à la première bande d'absorption. Une bande plus large avec un décalage Stokes beaucoup plus important n'apparaît pas sur la figure 2. Si on excite l'échantillon avec une impulsion picoseconde et si on résout temporellement cette luminescence, on observe que le pic étroit est une composante rapide (avec une constante de temps de l'ordre de la nanoseconde) alors que la bande large est une composante lente (la constante de temps se compte en dizaines ou centaines de microsecondes). Le pic étroit est dû à la recombinaison directe d'un électron dans le niveau $1 S_{e}$ avec un trou dans le niveau $1 S_{3 / 2}$. La bande large correspond à la recombinaison de porteurs piégés.

Ceci correspond à un point très important : la présence de pièges à la surface des nanocristaux. En fait, l'interface entre le nanocristal semi-conducteur et le diélectrique environnant est parfois assez mal contrôlée, surtout dans les verres. Dans ce dernier cas, le nombre de pièges est d'autant plus grand que le rayon des particules est plus petit. Les colloïdes permettent un meilleur contrôle de l'interface. En attachant des molécules organiques aux nanocristaux, on arrive à fortement réduire la bande large dans le spectre de luminescence et à obtenir un très bon rendement quantique de luminescence dans le pic étroit [13].

Autre point important : après excitation, le pic étroit et le bord « bleu » de la bande large apparaissent simultanément. On n'observe pas de croissance progressive de la bande large qui correspondrait au piégeage progressif des électrons. Ceci tend à montrer qu'il existe deux 


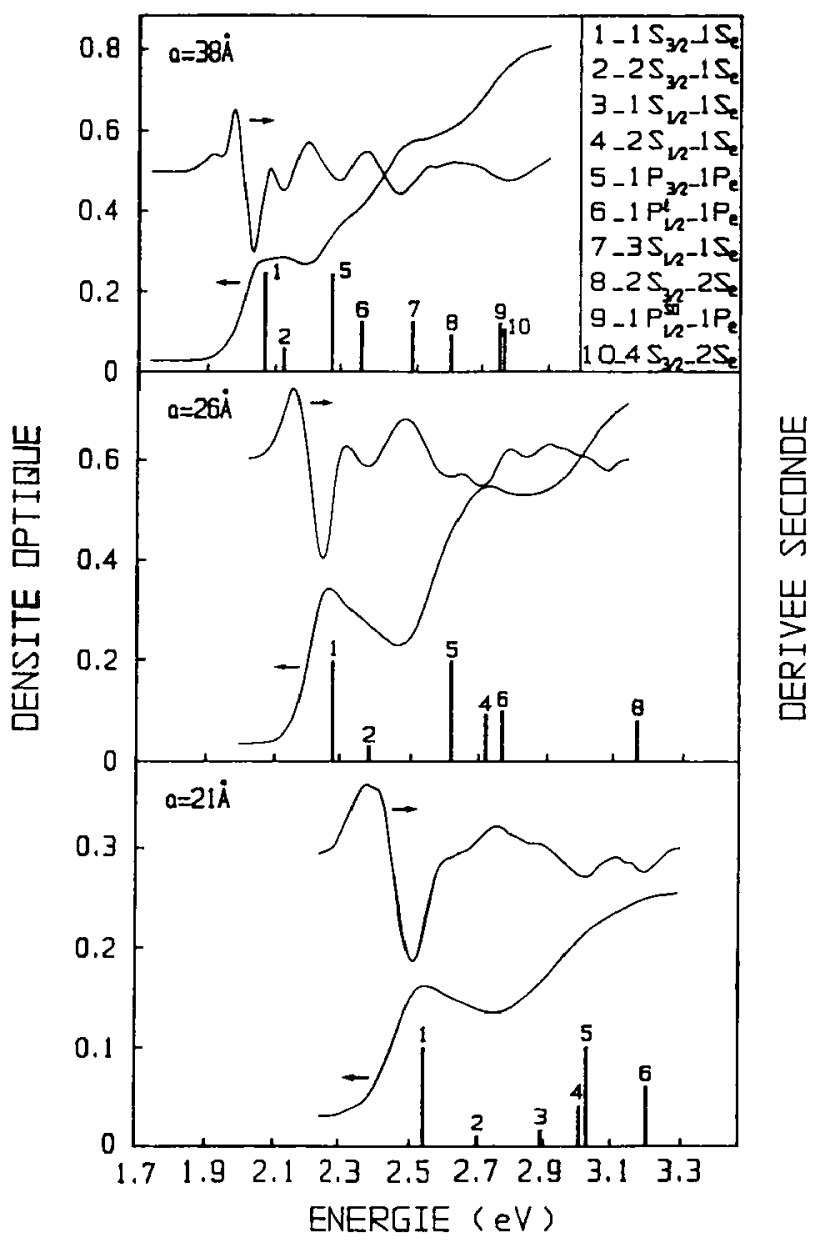

Figure 1 : Spectres d'absorption et leur dérivée seconde pour trois verres dopés par nanocristaux de CdSe. Le rayon moyen des particules est (de haut en bas) de $3.8 \mathrm{~nm}, 2.6 \mathrm{~nm}$ et $2.1 \mathrm{~nm}$.

classes de particules, des particules sans pièges qui donnent naissance au pic étroit et des particules avec pièges (dans lesquelles les électrons se piègent rapidement) à l'origine de la bande large [13].

\subsection{Photonoircissement}

Lorsqu'on irradie un verre dopé par nanocristaux semi-conducteurs par un faisceau laser intense, celui-ci subit un phénomène appelé photonoircissement [14] car dans certains cas une coloration grise apparaît. Ce phénomène a plusieurs autres conséquences. Le spectre de luminescence est fortement modifié. Le pic étroit est un peu moins intense qu'avant noircissement alors que la bande large a presque totalement disparu. Le temps de recombinaison des 


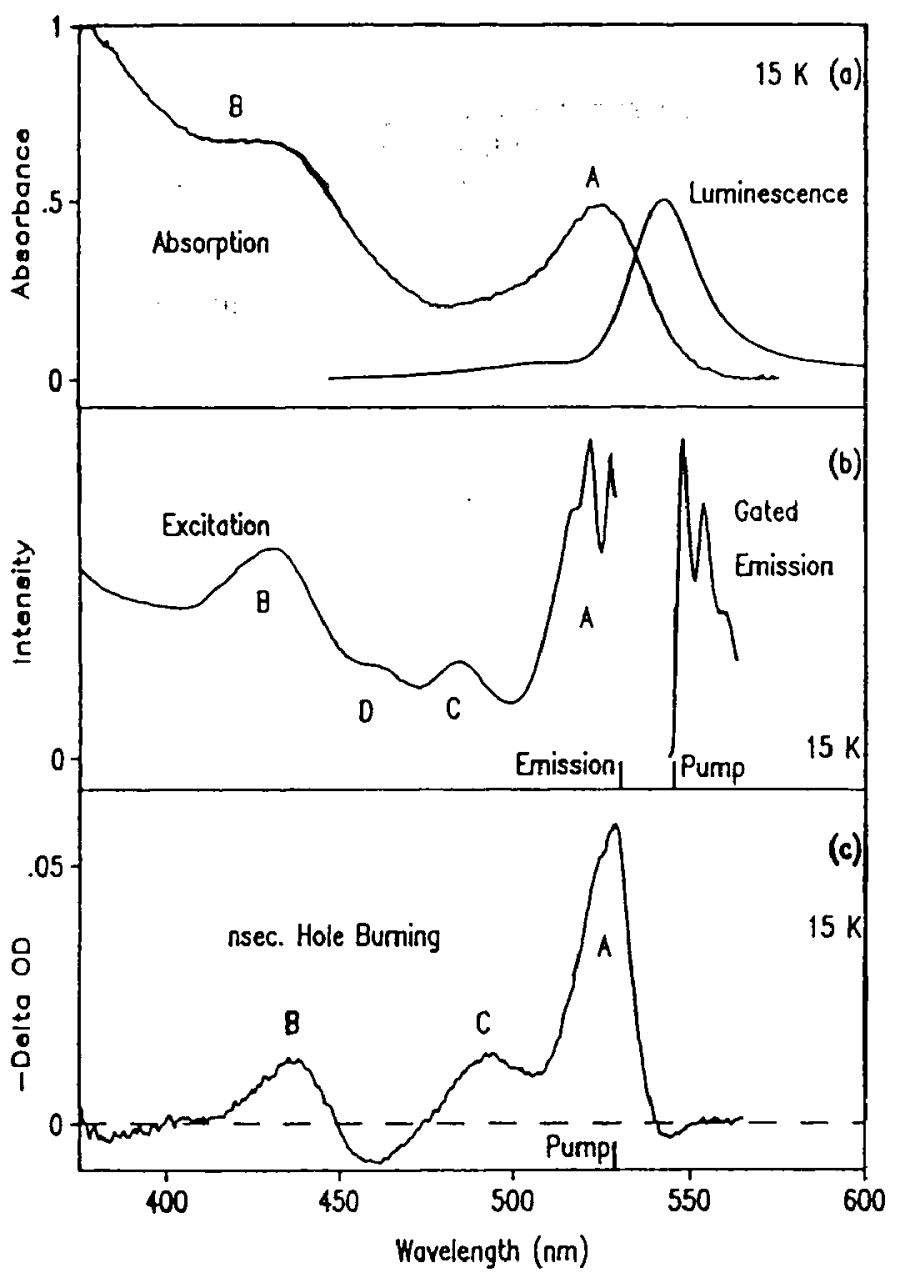

Figure 2 : Spectres d'absorption et de luminescence d'un collö̈de de CdSe : a) spectres lineaires, b) spectres PLE et FLN et c) spectre de hole-burning (d'après la réf. [12])

porteurs libres est plus court, ce qui explique la perte d'intensité du pic étroit. Nous verrons aussi que la non-linéarité d'un verre noirci est plus faible que celle d'un verre frais.

Le degré de noircissement ne dépend que de la dose d'irradiation (le produit intensité $\times$ durée) reçue. L'origine de l'effet a été élucidée par mesure de luminescence induite thermiquement [15]. Il s'agit d'une photo-ionisation ; un porteur photo-excité est éjecté du nanocristal et piégé dans la matrice de verre. Il s'agit d'un phénomène quasi permanent. Il peut être considéré comme permanent à température ambiante ou à basse température mais il est réversible par traitement thermique. En chauffant le verre à $-370^{\circ} \mathrm{C}$ pendant quelques heures, les porteurs piégés dans le verre diffusent vers leur nanocristal de départ et le verre retrouve ses propriétés originelles. 


\subsection{Largeur de raie et sous-structure}

Les spectres d'absorption et de luminescence des figures 1 et 2 a montrent clairement que les raies prévues par la théorie précédente sont fortement élargies. On connaît deux sortes d'élargissement : l'élargissement inhomogène et l'élargissement homogène. On devra aussi considérer la possibilité d'une sous-structure due à des degrés de liberté non encore pris en compte. Dans le cas des nanocristallites semi-conducteurs, l'élargissement inhomogène est surtout dû à la distribution de taille des particules. L'énergie des niveaux est fonction du rayon du nanocristal : un cristal plus petit absorbe plus vers le bleu, un cristal plus gros absorbe plus vers le rouge. Or, quelle que soit la méthode de préparation, tous les cristaux n'ont pas la même taille. Il en résulte donc une distribution des fréquences de transition. Cet élargissement inhomogène masque la largeur intrinsèque des raies.

Pour accéder à la largeur homogène, il faut avoir recours à des techniques de spectroscopie non linéaire. On peut par exemple exciter l'échantillon avec un rayonnement monochromatique (on excite alors sélectivement une classe de particules) et 1) regarder comment le spectre d'absorption a été modifié (c'est la technique du «hole burning ») ou 2) mesurer le spectre de luminescence (c'est la technique du «fluorescence line narrowing » ou FLN). Des exemples de résultats obtenus par les techniques 1) et 2) sont donnés au bas et au milieu de la figure 2 respectivement. Symétriquement au cas 2) ci-dessus, on peut sélectionner une tranche de fluorescence et mesurer son intensité en balayant la longueur d'onde d'excitation (c'est la technique de «photoluminescence excitation spectroscopy » ou PLE). Un exemple de spectre PLE est donné dans la partie intermédiaire de la figure 2. Pour tirer le maximum de la technique FLN, il faut exciter dans la queue rouge du spectre d'absorption. De même, pour tirer le maximum de la technique PLE, il faut regarder dans la queue bleue du spectre de luminescence.

En effet, la partie intermédiaire de la figure 2 montre que les spectres intrinsèques d'absorption ou d'émission ont une structure vibronique. Cette structure est due au couplage entre électrons et phonons LO dont la fréquence est de $210 \mathrm{~cm}^{-1}$ pour CdSe (nous n'avions pas tenu compte de ce degré de liberté de vibration) et est, elle aussi, masquée par l'élargissement inhomogène. Utilisée sur des colloïdes de CdSe, la technique PLE a permis de suivre en fonction de la taille 10 transitions électroniques et d'identifier les 6 premières [16]. Elle a aussi permis de mettre en évidence la structure fine du niveau $1 S_{3 / 2}-1 S_{e}$ qui est 8 fois dégénéré. La dégénérescence est en partie levée par la non-sphéricité des particules, par la structure cristalline et par l'interaction d'échange [17].

On peut également obtenir la largeur intrinsèque par des techniques d'écho de photons ou encore, travaillant sur un colloïde dilué et focalisant fortement le laser, isoler le spectre d'émission d'un seul nanocristal [18].

\section{PROPRIÉTÉS NON LINÉAIRES}

Les verres et les colloïdes étant isotropes, la non-linéarité qui nous intéresse est la non-linéarité d'ordre trois, essentiellement l'effet Kerr optique ou statique. L'effet Kerr statique correspond à la modification de la susceptibilité du milieu, proportionnellement au carré du champ électrique appliqué. L'effet Kerr optique généralise l'effet Kerr statique au cas d'un champ oscillant, la modification étant proportionnelle au module carré du champ laser. Ces propriétés peuvent être utiles pour les applications comme la commutation tout-optique ou dans des modulateurs dans le cas statique. 


\subsection{Effet Kerr statique}

On mesure en général le changement du spectre d'absorption induit par un champ statique ou basse fréquence. Ce changement est proportionnel à la partie imaginaire de la susceptibilité $\tilde{\chi}^{(3)}(0,0, \omega)$. Outre les applications potentielles, l'effet Kerr statique est intéressant en soi. Il est dû au déplacement des niveaux d'énergie et au mélange des fonctions d'onde non perturbées par le Hamiltonien de perturbation $\pm q$ E.r. Si l'espacement entre niveaux est grand devant la perturbation d'ordre qER comme c'est le cas pour les atomes, on peut traiter le problème par la théorie des perturbations. On parle alors d'effet Stark. Si au contraire les niveaux sont rapprochés, on doit diagonaliser le Hamiltonien total. On parle d'effet FranzKeldysh. L'effet physique est le même, seule l'approche mathématique diffère.

L'expérience a été faite sur un verre contenant des nanocristaux de $\mathrm{CdS}_{0.5} \mathrm{Se}_{0.5}$ [19]. Le champ électrique, avec $\mathrm{E}_{\mathrm{in}}=210^{4} \mathrm{~V} / \mathrm{cm}$, était appliqué grâce à des électrodes transparentes. C'était un champ sinusoïdal oscillant à $1 \mathrm{kHz}$. Le changement d'absorption était mesuré grâce à un amplificateur à détection synchrone. On observe alors des oscillations au voisinage de la bande $1 \mathrm{~s}-1 \mathrm{~s}$ avec une réplique au voisinage de la bande décalée par couplage spin-orbite. La position des oscillations est indépendante de la valeur du champ et leur amplitude est proportionnelle au carré du champ. Les résultats sont en bon accord avec un calcul de perturbations. On est donc en présence d'un effet Stark, l'espacement entre niveaux étant induit par le confinement. En augmentant la taille des particules, on observe la transition vers l'effet FranzKeldysh [20].

La partie imaginaire de $\tilde{\chi}^{(3)}(0,0, \omega)$ est de l'ordre de $10^{-20}(\mathrm{~m} / \mathrm{V})^{2}$ pour une fraction volumique d'environ $3 \mathbf{1 0}^{-3}$. La susceptibilité de la matrice hôte étant réelle, elle ne contribue pas à cet effet d'électro-absorption. Un champ statique conduit à une diminution de l'absorption à la fréquence du pic $1 \mathrm{~s}-1 \mathrm{~s}$ et à une augmentation de part et d'autre.

\subsection{Effet Kerr optique}

Dans ce cas, la fréquence laser peut coïncider avec une fréquence de transition, en général celle de la transition 1s-1s. On est alors en régime résonant et l'effet dominant est un effet de population ; des porteurs sont réellement créés et c'est leur présence qui modifie les propriétés optiques. La non-linéarité est alors grande, mais il y a des pertes par absorption et le temps de réponse $\tau$ est limité par la recombinaison des porteurs. Pour les applications, dans un tel cas, un matériau est caractérisé par le facteur de mérite $\chi^{(3)} / \alpha \tau$ où $\chi^{(3)}$ est la susceptibilité Kerr et $\alpha$ le coefficient d'absorption. En régime non résonant, par exemple pour des fréquences inférieures à la largeur de la bande interdite, l'effet dominant est le déplacement radiatif des niveaux. Le temps de réponse est très bref et, si $\mathrm{E}_{\mathrm{g}} / 2<\hbar \omega<\mathrm{E}_{\mathrm{g}}$, les seules pertes par absorption sont dues à l'absorption à deux photons liée à la partie imaginaire de $\chi^{(3)}$. Le facteur de mérite caractérisant le matériau est alors le rapport de la partie réelle de $\chi^{(3)}$ à sa partie imaginaire.

Différentes techniques ont été utilisées pour étudier l'effet Kerr optique : l'absorption non linéaire, la conjugaison de phase, le mélange de fréquence quasi dégénéré, la technique «Zscan ". Ces techniques donnent accès soit au module de $\tilde{\chi}^{(3)}\left(\omega_{1},-\omega_{1}, \omega_{2}\right)$, soit à ses parties réelle ou imaginaire. Un faisceau de fréquence $\omega_{1}$ modifie les propriétés optiques à la fréquence $\omega_{2}$. Dans le cas dégénéré, $\omega_{1}=\omega_{2}$. 


\subsubsection{Le régime résonant}

La population de l'état à une paire conduit à la saturation de la transition correspondante. Mais on peut créer une deuxième paire dans l'état $1 \mathrm{~s}-1 \mathrm{~s}$ et la même population conduit aussi à une absorption induite entre état à une paire et état à deux paires. Notons 0 l'état fondamental, 1 l'état à une paire et 2 l'état à deux paires. À cause de l'interaction coulombienne entre les paires, la fréquence de transition $\omega_{21}$ est légèrement inférieure à la fréquence de transition $\omega_{10}$ [21]. Supposant le même temps de déphasage $T_{2}$ pour les deux transitions et conservant uniquement les termes triplement résonants, la polarisabilité Kerr dans le cas dégénéré s'écrit [22]

$$
\begin{gathered}
\gamma(\omega,-\omega, \omega)=\frac{1}{\varepsilon_{0} \hbar^{3}}\left\{d_{10}^{4} \frac{T_{1} / T_{2}}{\left[\left(\omega-\omega_{10}\right)^{2}+1 / T_{2}^{2}\right]\left(\omega-\omega_{10}+i / T_{2}\right)}-\right. \\
-\frac{d_{21}^{2} d_{10}^{2}}{2}\left[\frac{T_{1} / T_{2}}{\left[\left(\omega-\omega_{10}\right)^{2}+1 / T_{2}^{2}\right]\left(\omega-\omega_{21}+\mathrm{i} / \mathrm{T}_{2}\right)}-\right. \\
\left.\left.-\frac{1 / 2}{\left(\omega-\omega_{10}+\mathrm{i} / \mathrm{T}_{2}\right)\left(2 \omega-\omega_{20}+\mathrm{i} / \mathrm{T}_{2}\right)}\left(\frac{1}{\omega-\omega_{21}+\mathrm{i} / \mathrm{T}_{2}}-\frac{1}{\omega-\omega_{10}+\mathrm{i} / \mathrm{T}_{2}}\right)\right]\right\}
\end{gathered}
$$

où $\mathrm{d}$ est l'opérateur moment dipolaire électrique et $\mathrm{T}_{1}$ la durée de vie du niveau 1. $\gamma$ est la somme de trois termes. Le premier correspond à la saturation de la transition $0 \rightarrow 1$, le second à l'absorption induite $1 \rightarrow 2$. Le troisième terme qui est une contribution cohérente est négligeable car $T_{2} \ll T_{1}$. Les deux termes importants, saturation de l'absorption et absorption induite, constituent la contribution des porteurs libres.

Nous savons qu'il existe aussi des porteurs piégés à l'interface. Ces porteurs piégés, par l'intermédiaire du champ statique qu'ils créent, modifient les propriétés optiques par effet Kerr statique. Les expériences étant réalisées avec des impulsions picoseconde dont la durée est courte devant la durée de vie du niveau 1 ou des porteurs piégés, il faut également tenir compte de l'aspect transitoire de la réponse. On utilise pour cela le formalisme de la fonction réponse. On peut aussi introduire la notion de susceptibilité effective. Le calcul peut être fait simplement dans le cas d'impulsions rectangulaires [23].

Utilisant la technique pompe-sonde, la saturation de l'absorption a été observée de même que l'absorption induite. La réponse non linéaire peut être résolue temporellement. On observe qu'elle est en général faite d'une composante rapide et d'une composante lente comme le montre la figure 3. En travaillant sur un verre dopé par nanocristaux de $\mathrm{CdS}_{0.3} \mathrm{Se}_{0.7}$ noirci à des degrés divers par un faisceau à profil gaussien, on constate que, pour la composante rapide, la constante de temps décroît avec le degré de noircissement alors que l'amplitude reste constante. L'amplitude de la composante lente décroît avec le degré de noircissement jusqu'à s'annuler. Ces résultats sont en accord avec le modèle précédent [23]. Ils confirment de plus l'hypothèse des deux classes de particules, les particules sans pièges donnant naissance à la composante rapide (contribution des porteurs libres) et les particules avec pièges à la composante lente. Tout se passe comme si les particules noircies ne contribuaient plus à la non-linéarité. 


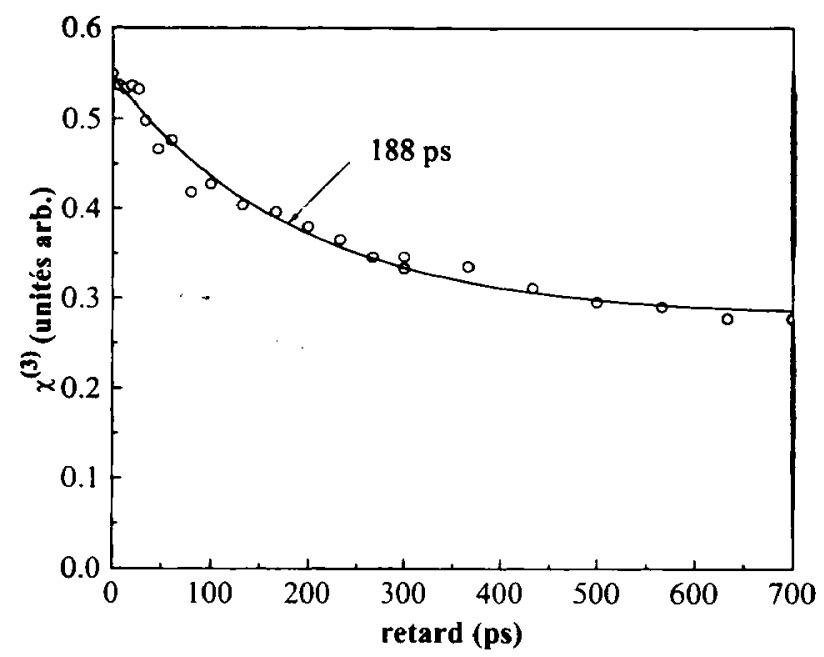

Figure 3 : Susceptibilité Kerr effective en fonction du retard de l'impulsion sonde pour un verre modérément noirci.

Pour un échantillon frais, la composante lente domine généralement alors que, pour un échantillon noirci, c'est la composante rapide qui domine. Le mécanisme dominant ne peut pas être prévu a priori; il dépend de l'origine et de l'histoire de l'échantillon. Utilisant un milieu référence tel que $\mathrm{CS}_{2}$, on peut mesurer la grandeur de la susceptibilité effective (composantes rapide et lente). La grandeur de la composante lente qui dépend bien sûr de la situation est de l'ordre de $10^{-18}(\mathrm{~m} / \mathrm{V})^{2}$ et en bon accord avec les prévisions théoriques. Celle de la composante rapide qui est du même ordre indique une certaine compensation entre les effets de saturation de l'absorption et d'absorption induite. Le facteur de mérite défini cidessus est inférieur à celui de la non-linéarité excitonique des puits quantiques. Cela est dû à la grande largeur inhomogène de la bande $1 \mathrm{~s}-1 \mathrm{~s}$. Il serait intéressant d'étudier des échantillons pour lesquels la distribution de taille est plus étroite.

Pour un échantillon noirci, ne subsiste que la composante rapide. On observe alors que le facteur de mérite diminue lorsque la taille des nanocristaux diminue [24]. Rappelons qu'il fait intervenir le temps de recombinaison des porteurs libres qui peut être fortement réduit par le noircissement. C'est ainsi qu'un dispositif bistable avec un temps de réponse de 25 picosecondes a pu être fabriqué avec ce genre de matériau [25].

\subsubsection{Le régime non résonant}

Nous considérons maintenant le cas où le faisceau laser n'est pas absorbé. Pour le semiconducteur massif, nous dirions $\hbar \omega<\mathrm{E}_{\mathrm{g}}$. Certains auteurs définissent une largeur de bande interdite $E_{g}$ effective qui tient compte du confinement. $\chi^{(3)}(\omega,-\omega, \omega)$ n'est plus triplement résonant mais, si $\mathrm{E}_{\mathrm{g}} / 2<\hbar \omega<\mathrm{E}_{\mathrm{g}}$, il existe encore une résonance à deux photons $\omega+\omega$ qui conduit au phénomène d'absorption à deux photons. En régime non résonant, c'est cette 
absorption à deux photons qui a d'abord été étudiée. Dans ce cas, l'intensité I du faisceau laser est atténuée selon l'équation

$$
\frac{d I}{d z}=-\beta I^{2}-N \sigma I
$$

où $\beta$, proportionnel à la partie imaginaire de $\tilde{\chi}^{(3)}(\omega,-\omega, \omega)$, est le coefficient d'aborption à deux photons du verre dopé. Le deuxième terme correspond à l'absorption des porteurs libres créés par absorption à deux photons. $\mathrm{N}$ est le nombre de porteurs libres par unité de volume et est donné par

$$
N(t)=\int_{-\infty}^{t} \frac{\beta I^{2}\left(t^{\prime}\right)}{2 \hbar \omega} d t^{\prime}
$$

en négligeant la recombinaison des porteurs à l'échelle de la picoseconde. $\sigma$ est la section efficace d'absorption des porteurs libres.

Les règles de sélection sont différentes pour l'absorption à deux photons et pour l'absorption linéaire [26]. Les valeurs des paramètres $\beta$ et $\sigma$ ont été déduites de mesures de la transmission en fonction de l'intensité incidente. Pour des impulsions picoseconde, l'absorption des porteurs libres est non négligeable. La matrice ne contribue pas à l'absorption à deux photons. À partir de la susceptibilité $\tilde{\chi}^{(3)}$ du composite, connaissant la fraction volumique $p$, on peut en déduire la partie imaginaire de la susceptibilité $\chi^{(3)}$ d'un nanocristal.

La réfraction non linéaire a aussi été étudiée dans ce régime de fréquence. Pour éviter la contribution des porteurs libres, la technique du mélange de fréquence a été utilisée. On en déduit la valeur de la partie réelle de $\tilde{\chi}^{(3)}$. Ici, la contribution de la matrice $\chi_{d}^{(3)}$ est importante. En en tenant compte, on peut obtenir la partie réelle de $\chi^{(3)}$. Certains auteurs observent que la susceptibilité du nanocristal, partie imaginaire et partie réelle, n'est pas très différente de celle du semi-conducteur massif [27,28]. Mais les échantillons étudiés n'avaient pas un spectre d'absorption très structuré et le rôle du confinement n'est pas encore clair.

\section{CONCLUSION}

Le confinement électronique conduit à un spectre de raies pour un nanocristal semi-conducteur. Mais ces raies sont souvent fortement élargies à cause en particulier de la distribution de taille. Les propriétés non linéaires ont été étudiées en assez grand détail, surtout en régime résonant. Le facteur de mérite est dans ce cas inférieur à celui des puits quantiques. Ceci est en grande partie dô à la polydispersité des échantillons étudiés. Prenant l'exemple de l'action d'un champ statique, le pic $1 S_{3 / 2}-1 S_{e}$ est l'analogue d'un pic excitonique tel qu'on en trouve dans les puits quantiques, mais l'élargissement inhomogène est beaucoup plus important. Pour ce qui concerne le régime non résonant, les études doivent être poursuivies sur des particules à confinement plus fortement marqué.

L'avenir des boîtes quantiques dépend donc de notre capacité à mieux contrôler leur fabrication du point de vue de la monodispersité mais aussi du point de vue du nombre de pièges. Des progrès ont déjà été réalisés. Les nanocristaux de semi-conducteurs III-V pour lesquels il 
est plus facile d'atteindre le régime de confinement fort devraient aussi s'avérer extrêmement prometteurs.

\section{Références}

[1] Böttcher C.J., Theory of Electric Polarization, (Elsevier, Amsterdam, 1973) p. 78.

[2] Kreibig U. and Zacharias P., Z. Phys., 231 (1970) 128-143.

[3] Neuendorf R., Quinten M. and Kreibig U., J. Chem. Phys., 104 (1996) 6348-6354.

[4] Efros Al.L. and Efros A.L., Sov. Phys. Semicond., 16 (1982) 772-775.

[5] Ekimov A.I. and Efros Al.L., phys. stat. sol. (b), 150 (1988) 627-633.

[6] Efros Al.L. and Rodina A.V., Phys. Rev. B, 47 (1993) 10005-10007.

[7] Ekimov A.I., Hache F., Schanne-Klein M.C., Ricard D., Flytzanis C., Kudryavtsev I.A., Yazeva T.V., Rodina A.V. and Efros Al.L., J. Opt. Soc. Am. B, 10 (1993) 100-107.

[8] Brus L.E., J. Chem. Phys., 80 (1984) 4403-4409.

[9] Banyai L., Gilliot P., Hu Y.Z. and Koch S.W., Phys. Rev. B, 45 (1992) 14136-14142.

[10] Lippens P.E. and Lannoo M., Phys. Rev. B, 41 (1990) 6079-6081.

[11] Luttinger J.M. and Kohn W., Phys. Rev., 97 (1955) 869-883.

[12] Bawendi M.G., Wilson W.L., Rothberg L., Carroll P.J., Jedju T.M., Steigerwald M.L. and Brus L.E., Phys. Rev. Lett., 65 (1990) 1623-1626.

[13] Bawendi M.G., Carroll P.J., Wilson W.L. and Brus L.E., J. Chem. Phys., 96 (1992) 946-954.

[14] Roussignol P., Ricard D., Lukasik J. and Flytzanis C., J. Opt. Soc. Am. B, 4 (1987) 513.

[15] Grabovskis V.Ya., Dzenis Ya.Ya., Ekimov A.I., Kudryavtsev I.A., Tolstoï M.N. and Rogulis U.T., Sov. Phys. Solid State, 31 (1989) 149-151.

[16] Norris D.J. and Bawendi M.G., Phys. Rev. B, 53 (1996) 16338-16346.

[17] Efros Al.L., Rosen M., Kuno M., Nirmal M., Norris D.J. and Bawendi M.G., Phys Rev B, 54 (1996) 4843-4856.

[18] Empedocles S.A., Norris D.J. and Bawendi M.G., Phys. Rev. Lett., 77 (1996) 3873-3876

[19] Hache F., Ricard D. and Flytzanis C., Appl. Phys. Lett., 55 (1989) 1504-1506.

[20] Cotter D., Girdlestone H.P. and Moulding K., Appl. Phys. Lett., 58 (1991) 1455-1457.

[21] Hu Y.Z., Koch S.W., Lindberg M., Peyghambarian N., Pollock E.L. and Abraham F.F., Phys. Rev. Lett., 64 (1990) 1805-1807.

[22] Banyai L., Hu Y.Z., Lindberg M. and Koch S.W., Phys. Rev. B, 38 (1988) 8142-8153.

[23] Ghanassi M., Piveteau L., Saviot L., Schanne-Klein M.C., Ricard D. and Flytzanis C., Appl. Phys. B, 61 (1995) 17-26.

[24] Schanne-Klein M.C., Piveteau L., Ghanassi M. and Ricard D., Appl. Phys. Lett., 67 (1995) 579-581.

[25] Yumoto J., Fukushima S. and Kubodera K., Optics Lett., 12 (1987) 832-834.

[26] Kang K.I., McGinnis B.P., Sandalphon, Hu Y.Z., Koch S.W., Peyghambarian N., Mysyrowicz A., Liu L.C. and Risbud S.H., Phys. Rev. B, 45 (1992) 3465-3468.

[27] Banfi G.P., Degiorgio V., Ghigliazza M., Tan H.M. and Tomaselli A., Phys. Rev. B, 50 (1994) 5699-5702.

[28] Banfi G.P., Degiorgio V., Fortusini D. and Tan H.M., Appl. Phys. Lett., 67 (1995) 13-15 